\title{
Review Paper \\ Medicinal Plants Effective in the Prevention and Control of Coronaviruses
}

\author{
*Saghar Ketabchi ${ }^{1}[10$, Maryam Papari Moghadamfard² [
}

1. Department of Plant Pathology and Plant Protection (Microbiology), Shiraz Branch, Islamic Azad University, Shiraz, Iran 2. Department of Chemistry, Shiraz Branch, Islamic Azad University, Shiraz, Iran.

\begin{tabular}{l|l}
$\begin{array}{c}\text { Use your device to scan } \\
\text { and read the article online }\end{array}$ \\
Cftation: Ketabchi S, Papari Moghadamfard M. [Medicinal Plants Effective in the Prevention and Control of Coronaviruses \\
(Persian)]. Complementary Medicine Journal. 2021; 10(4):296-307. https://doi.org/10.32598/cmja.10.4.1014.1
\end{tabular}

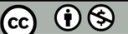

Article Info:

Received: 20 Aug 2020

Accepted: 07 Nov 2020

Available Online: 01 Jan 202

Key words:

COVID-19, SARS,

MERS, Natural prod-

uct, Phytotherapy

\section{AB STRACT}

Objective Coronaviruses often cause acute complications in the respiratory system with cold-like symptoms. A number of them, such as Severe Acute Respiratory Syndrome (SARS) and Middle East Respiratory Syndrome (MERS) and Coronavirus Disease 2019 (COVID-19) have killed thousands of people and have caused epidemics and pandemics. This review study aims to investigate the most common medicinal plants in Iran and introduce their natural products with antiviral effects on coronaviruses and strengthening the immune system in order to prevent and control them.

Methods In this review study, a search was conducted in national and international databases such as Web of Science, Scopus, PubMed, Science Direct, Google Scholar, SID, Maglran and IranMedex by using keywords such as COVID-19, Coronaviruses, SARS, MERS, SARS-CoV-2, PEDV in both Persian and English for studies published until 2020, and finally 51 articles were selected.

Results There are 10 plants with antiviral effects on members of the family Coronaviridae among which Ginger, Galangal, Cinnamon, Fennel flower, Grapefruit (peel), and Purple coneflower were effective on COVID-19. Elder, Ginseng, Aloe vera, Milkvetch, and Shirazi Thyme plants were effective in boosting the immune system and preventing viral diseases.

Conclusion Inhibiting the replication of viruses is the common mechanism in antiviral drugs, but natural compounds usually counteract it by disrupting key proteins and virulence factors of viruses. Therefore, the use of the antiviral components of reported plants can be useful in producing drugs for these viruses, especially the one causing COVID-19.

\section{Extended Abstract}

\section{Introduction}

oronaviruses are a group of related RNA viruses that causes acute complications in the respiratory system so similar to cold symptoms. A number of them like Severe Acute Respiratory Syndrome (SARS) and Middle East Respiratory Syndrome (MERS) have caused epidem- ics with high mortality rate. Coronaviruses were first identified in 1965 and named as B814. So far, this virus has spread to birds, mammals and humans. In 2003, the World Health Organization identified a deadly infectious disease and named it SARS. Later, different types of Coronavirus were identified and introduced including Human Coronavirus NL63 in 2004, Hokovirus (HKV1) discovered in patients with pneumonia, MERS in 2012, and Porcine Epidemic Diarrhea Virus (PEDV) in 2014. The new type of these viruses that has caused a disease named "COVID-19" and is re-

\section{"Corresponding Author:}

Saghar Ketabchi, PhD.

Address: Department of Plant Pathology and Plant Protection (Microbiology), Shiraz Branch, Islamic Azad University, Shiraz, Iran.

Tel: +98 (713) 6410040

E-mail: ketabchis@gmail.com 
sponsible for the current pandemic was identified in Wuhan, China in 2019. In recent years, the use of herbal plants has been considered due to their less side effects and natural origin compared to chemical drugs, having anti-inflammatory, antimicrobial, anticonvulsant and antipyretic properties, and having compounds such as polyphenols and monophenols. This study aims to review the plants with inhibitory and antiviral effects on the Coronavirus family and also with the power to strengthen the immune system to prevent diseases.

\section{Material and Methods}

In this review study, the search was conducted in Web of Science, Scopus, PubMed, Science Direct, Google Scholar, SID, MagIran and IranMedex databases for related studies conducted until 2020 using the keywords such as Medicinal plants, COVID-19, Coronaviruses, SARS, MERS, SARS-CoV-2, and PEDV. First the abstracts of the articles were examined and the related studies that were thematically related to the objectives of the current study were then selected. Among these articles, those that included the definition of coronaviruses, the effects of plant extracts and essential oils on coronaviruses, and the therapeutic and inhibitory role of these medicinal plants on these viruses were selected. After finding effective plants in the treatment and prevention of coronaviruses, new search in above databases was conducted on them using the keywords: Essential oil, extract, effective compounds, phytochemical properties and chemical compounds; and their botanical characteristics and effective compounds were determined. Then, the effective compounds identified in the articles were matched with the compounds mentioned in the articles that reported the therapeutic and preventive effects of plants on Coronavirus, and the reasons for their antiviral nature were determined. The article were divided into three group: 1 . Articles related to definition and characteristics of Coronaviruses; 2 . Articles related to the effect of medicinal plants on Coronavirus; and 3. Articles related to the analysis of essential oils and plant extracts, chemical properties of effective compounds, and their mechanism of action. A total of 250 articles were yielded. Of these, 51 (9 in Persian and 42 in English) were selected for review.

\section{Results}

Studies have shown that 10 plants have an antiviral effect on the members of the family Coronaviridae including Ginger (Zingiber officinale), Galangal (Alpinia officinarum), Cinnamon (Cinnamomum zeylanicum), Fennel flower (Nigella sativa L.), Grapefruit (Citrus aurantium), Purple coneflower (Echinacea angustifolia), baylaure (Laurus nobilis), Mugwort (Artemisia sp.), Ginseng (Panax quinquefollus) and Liquorice (Glycyrrhiza glabra L.). These plants are effective treatment of SARS, MERS, Respiratory Syncytial Virus (RSV) and COVID-19. Elder (Sambucus nigra), Ginger, Aloe Vera, Milkvetch (Astragalus membranaceus), and Shirazi thyme (Zataria Multiflora) plants have been introduced as an immune system booster and as an effective factor in preventing viral diseases. These plants can somehow inhibit the activity of Coronaviruses by having various effective compounds. Due to the existence of glycyrrhizic acid composition in Liquorice, effective combination of lycorine in Mugwort, Flavonoids in baylaure, and hetero polysaccharides purple coneflower, these plants can have positive effects on SARS. On the other hand, Ginger can positively affect RSV due to having Ginsenoside compounds. Moreover, Ginger (due to existence of Phenol compounds such as Gingerol, Shogaol), Galangal (due to existence of Flavonoids compounds), Cinnamon (due to existence of Eugenol compounds), Purple coneflower (due to existence of Niglidin and Alpha hydrogen compounds) and skin of Grapefruit (due to existence Flavonoids compounds) have effects on COVID-19. It seems that each of the plants mentioned above show their antiviral activity by binding to the viral protease and preventing virus replication. Since the risk of developing Coronaviruses increases by weakening of the immune system, some plants have been introduced for strengthening the immune system including: Elder due to having Flavonoids, Ginger due to having phenolic and ginsenoside compounds, Aloe Vera due to having amino acid, Milkvetch due to having glucuronic acid, and Shirazi thyme due to having thymol and carvacrol contents.

\section{Conclusion}

It can be concluded that plants with effective compounds can be effective in inhibiting Coronaviruses. Five plants for this purpose were reported in this study. These plants boost the immune system against viral diseases by some mechanisms such as increase of cytokines by monocytes, induction of interferon and phagocyte production, increase of intestinal probiotic bacteria, induction of immunoglobulins and lymphocytes, protecting the central nervous system against viral infections, neutralizing hydrogen peroxide and oxygen free radicals, and reduction of cell death caused by system weakness. Inhibiting the replication of viruses is one of the common approaches in antiviral drugs, but natural compounds usually counteract it by disrupting key proteins and virulence factors of viruses. Therefore, the use of the effective antiviral compounds of these plants can be useful in producing drugs against these viruses causing diseases such as COVID-19 that has killed thousands of people and overshadowed the world economy and international relations. 


\section{Ethical Considerations}

Compliance with ethical guidelines

There were no ethical considerations to be considered in this research.

Funding

This research did not receive any grant from funding agencies in the public, commercial, or non-profit sectors.

Authors' contributions

All authors contributed in preparing this paper.

Conflicts of interest

The authors declared no conflict of interest.

Acknowledgements

The authors would like to thank Islamic Azad University, Shiraz Branch. 


\title{
كياهان دارويي مؤثر در بيشخيرى و كنترل كروناويروسها
}

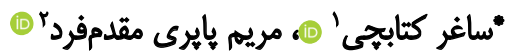

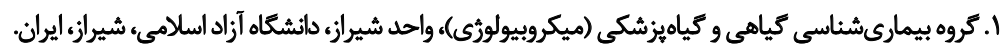

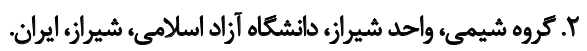

\begin{abstract}
سيكيد

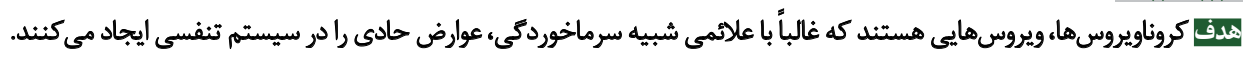

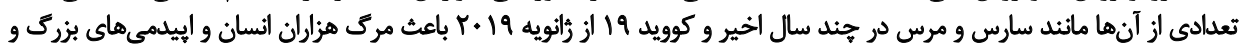

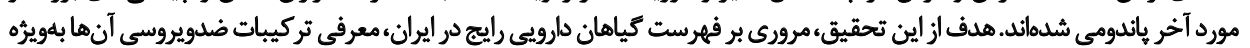

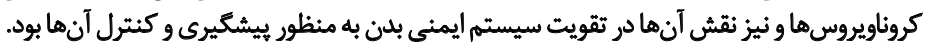

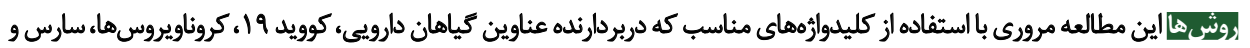

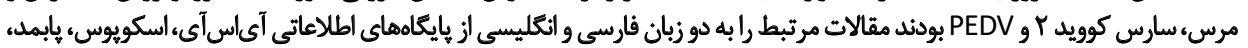

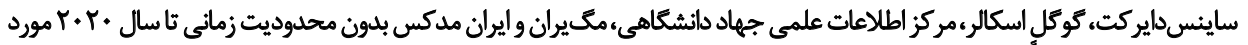

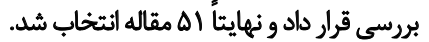

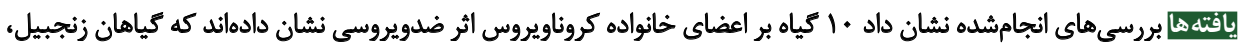

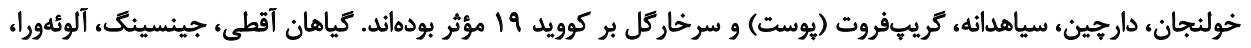

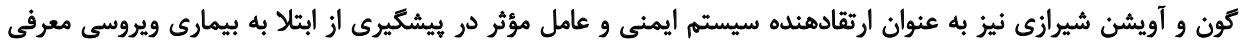
شدهاند.

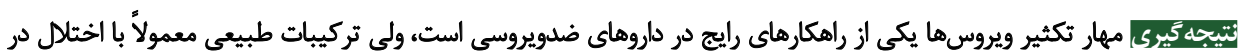

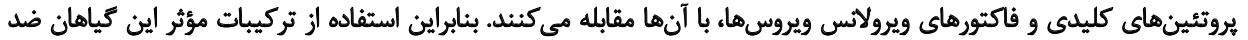

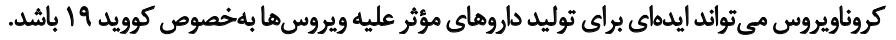

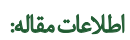

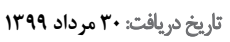

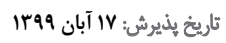

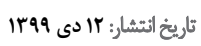

كليدوازوها:

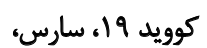
مرسي، تركيبات طبيعي،

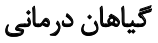

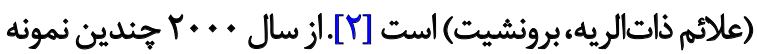

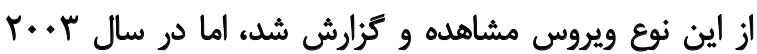

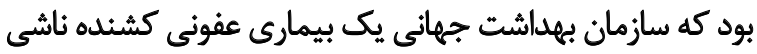

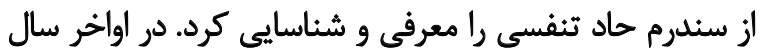

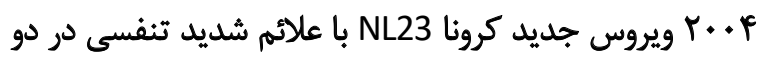

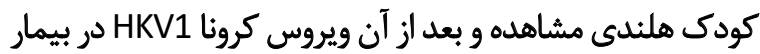

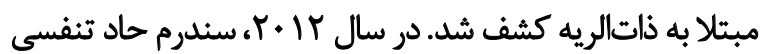

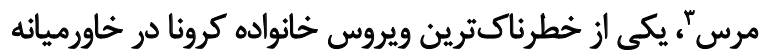

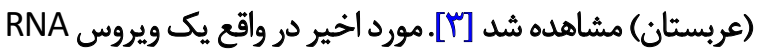

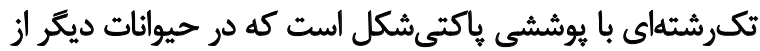

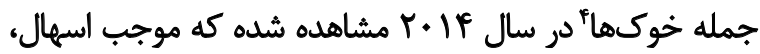

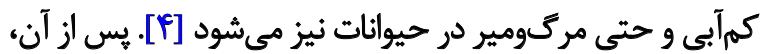

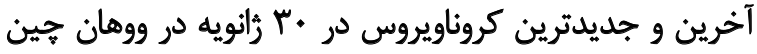

3. MERS-COV

4. Porcine Epidemic Diarrhea Virus (PEDV)
مقلمه كروناويروس براى اولينبار در سال 1990 توسط تيرل و باينو'

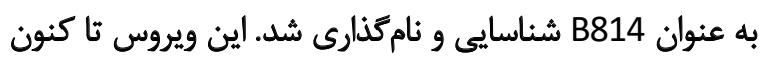

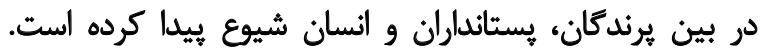

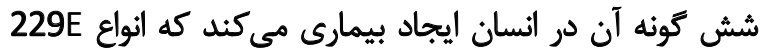

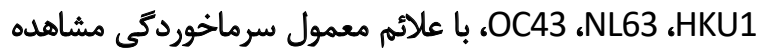

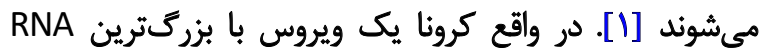

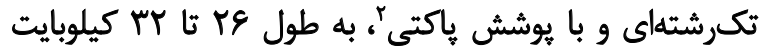

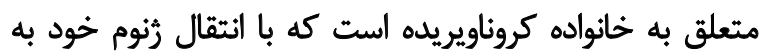

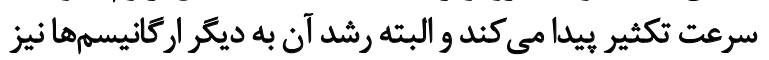

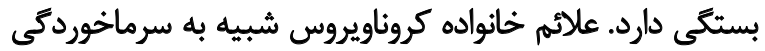

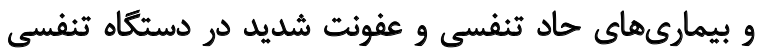

1. Tyrrel \& Bynoe

2. Envelope 
كرفتند كه اثر ضدويروسى اين تركيبات مشاهده شد و از آن بآن ئن

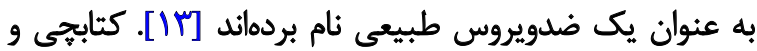

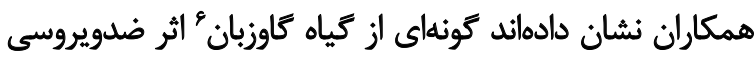

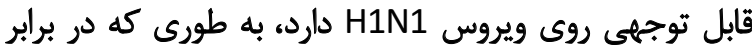

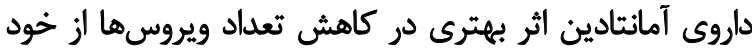

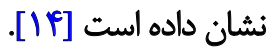

هدف اين تحقيق، مرورى بر فهرست كياهانى است كه داراي

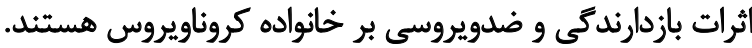

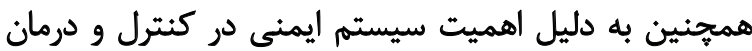

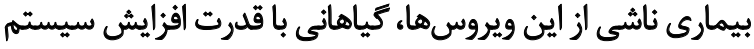
ايمنى به منظور بيشكيرى نيز مورد بررسى قرار كرفتند.

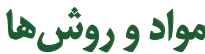

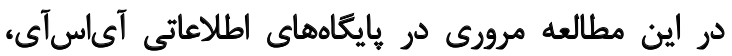

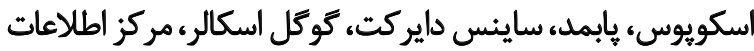

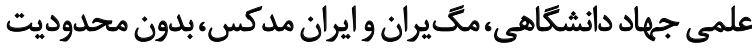

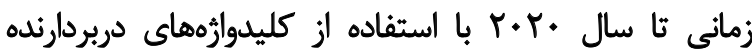

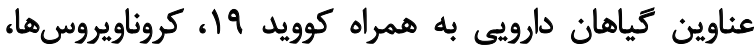

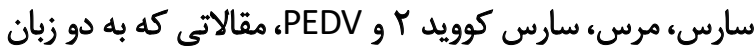

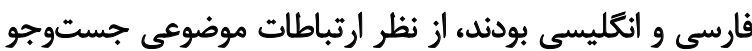

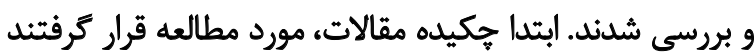

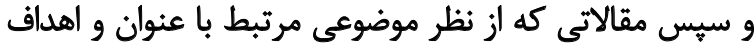

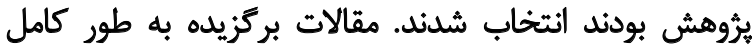

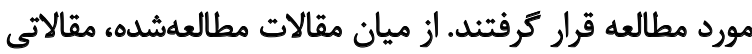

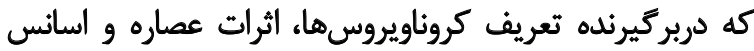

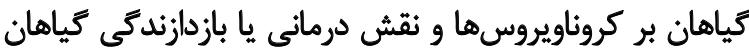

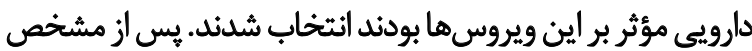

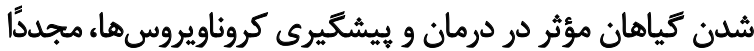

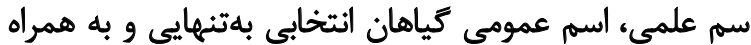

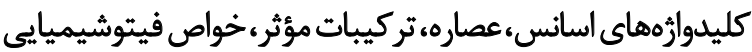

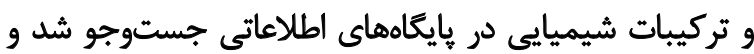

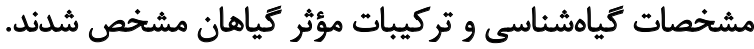

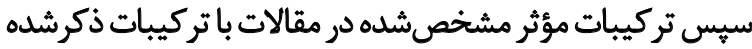

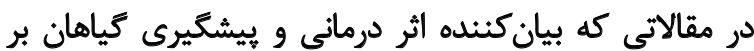

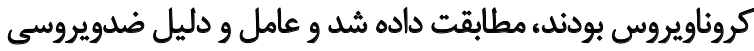

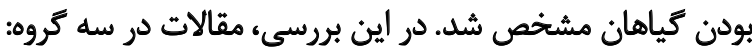

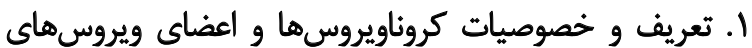

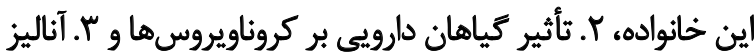
اسانس و عصاره كياهان، خصوصيات شيمياييى تركيبات مؤثره و

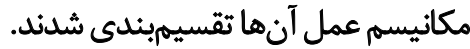

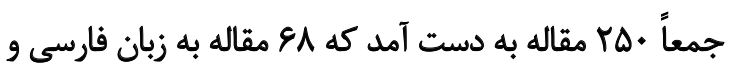

\section{Anchusa italicum}

كويدار شد كه سازمان بهداشت جهانى آن را كوويد 19 (بيماري

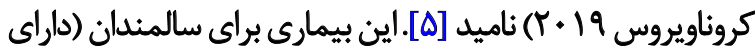

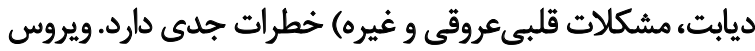

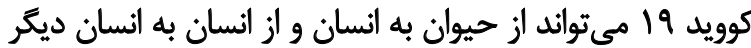

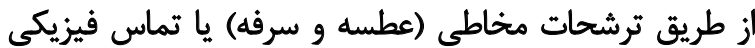

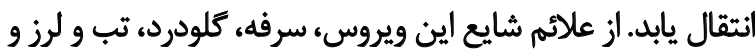

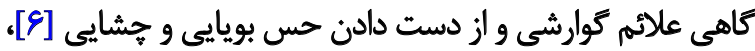

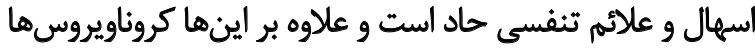

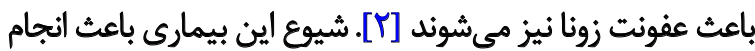

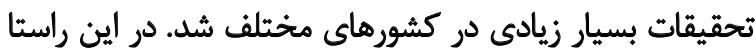

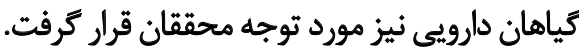

كياهان دارويى به دليل منشأ طبيعى خود، داراى عوارض جانبى كمترى نسبت به مواد شيميايى هستئد. امروزه كياهي داريان

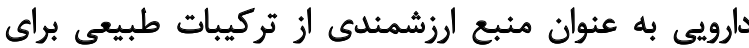

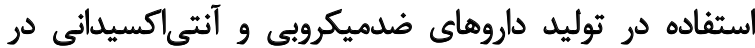

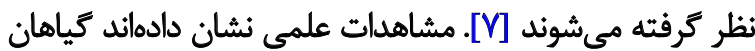

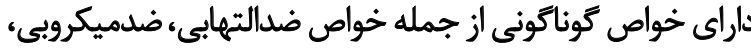

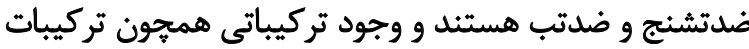

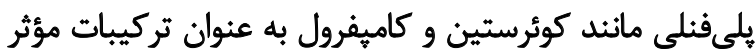

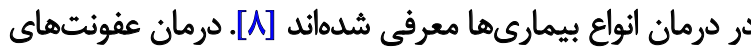

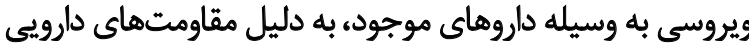

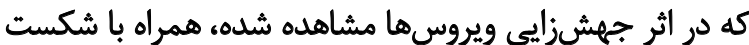

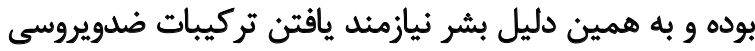

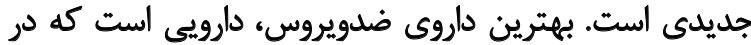

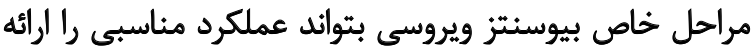

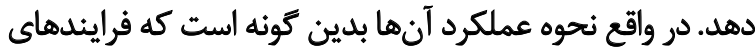

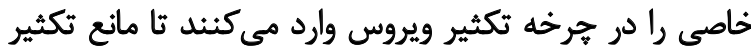

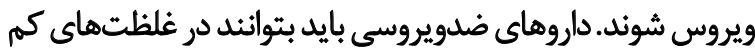
اثر خود را بكذارند [9].

در اين راستا، دانشمندان با مشاهده اثر تركيبات فرار كياهان

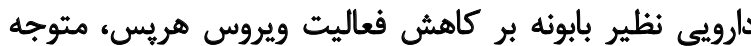

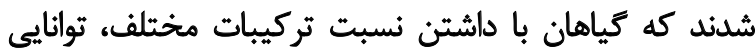

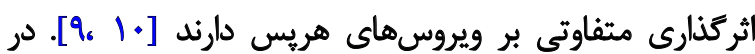

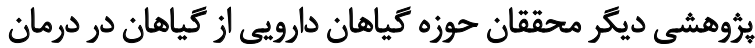

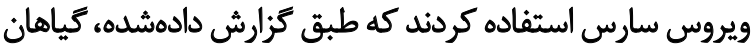

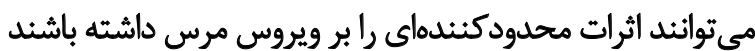

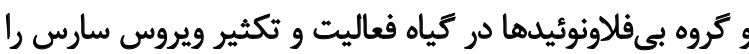

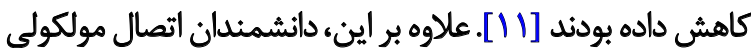

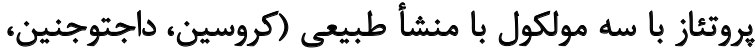

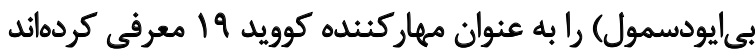

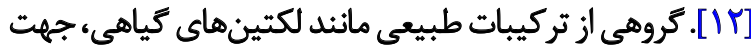

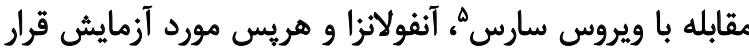

5. Severe Acute Respiratory Syndrome (SARS) 
درمنه

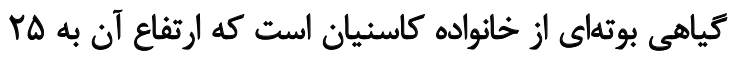

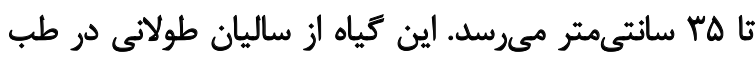

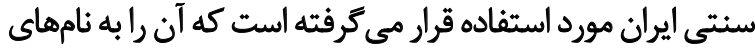

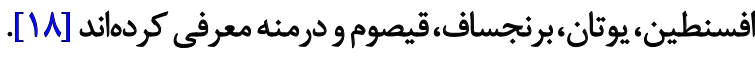

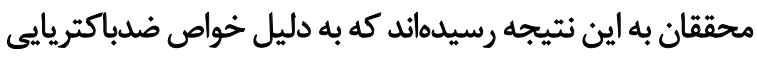

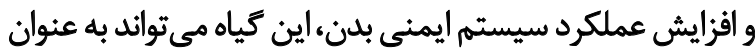

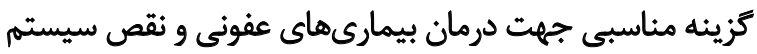

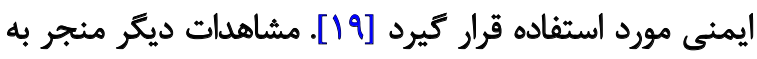

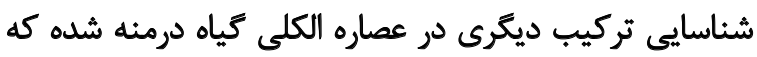

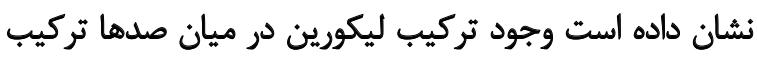

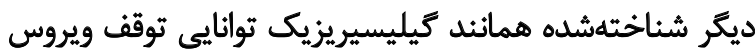

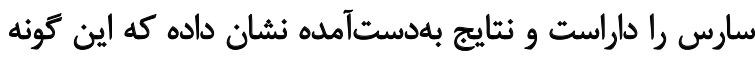

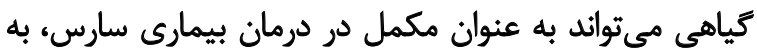

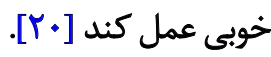

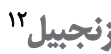

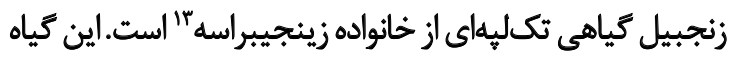

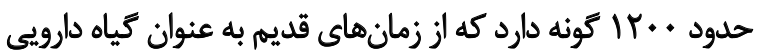

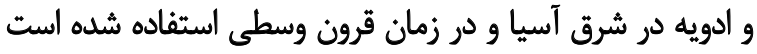

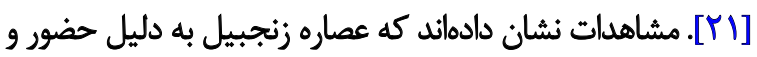

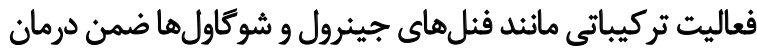

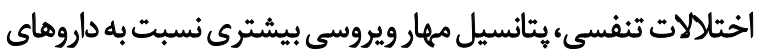

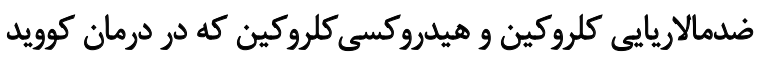

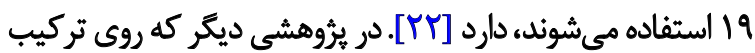

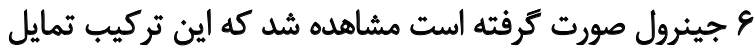

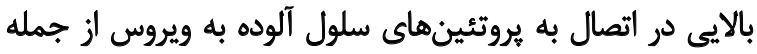

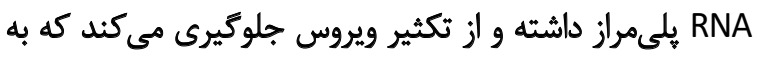

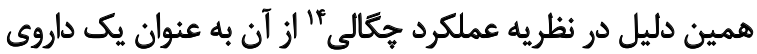

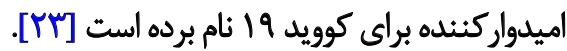

\section{خولئجان 18}

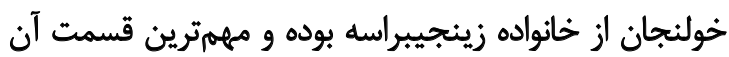

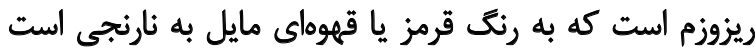

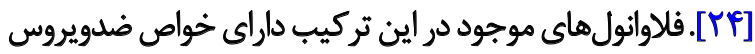

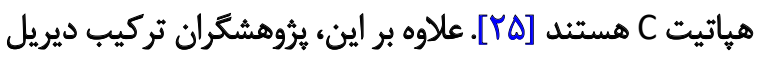

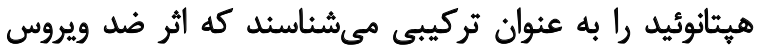

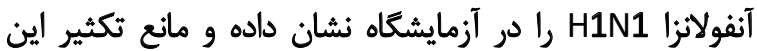

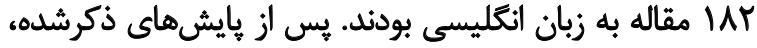

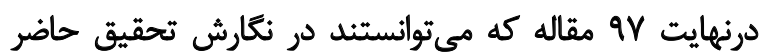

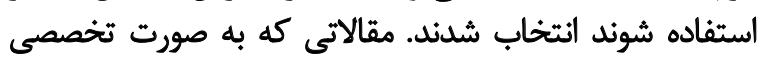

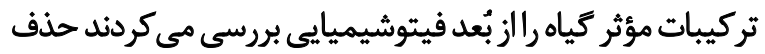

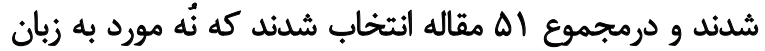
فارسى و Fr مورد به زبان انتليسى بودند.

يافتهها

\section{كياهان با اثر ضدويروسى بر كروناويروسها}

فهرست كياهان مؤثر بر كروناويروسها در جدول شماره آ آورده

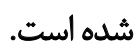

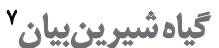

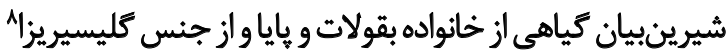

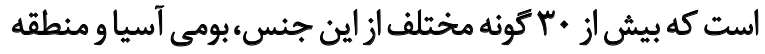

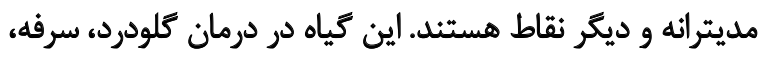

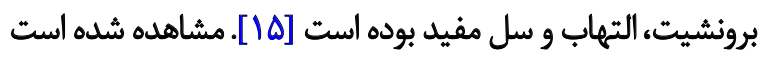

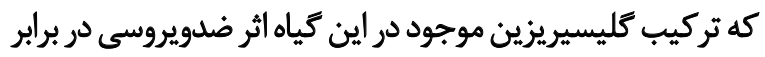

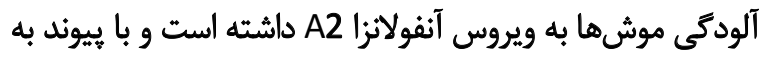

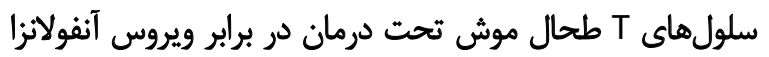

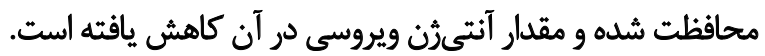

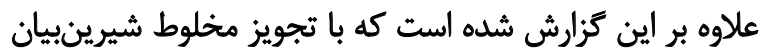

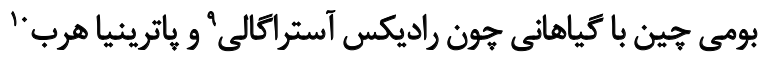

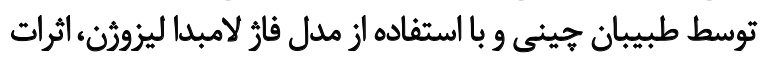

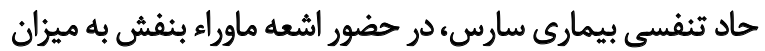

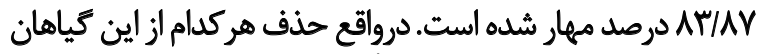

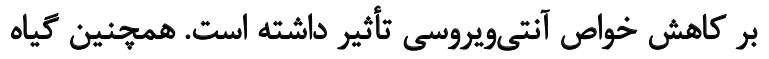

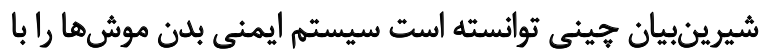

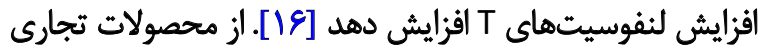

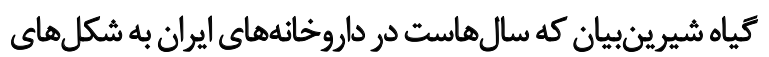

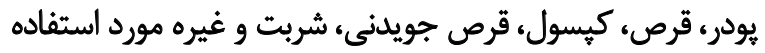

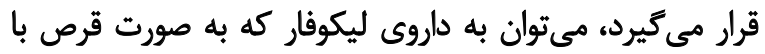

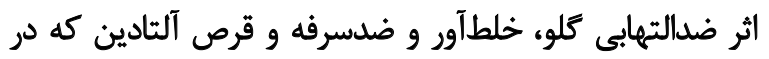

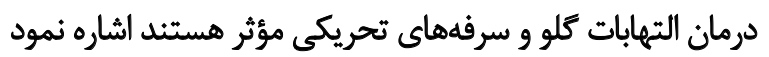

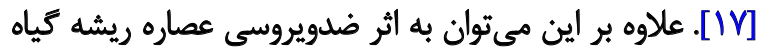

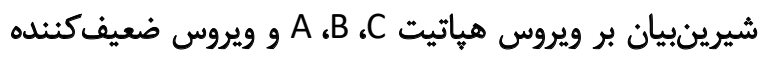

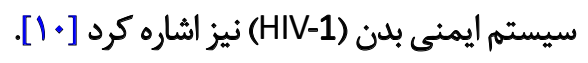

11. Mugworts (Artemisia sp.)

12. Ginger (Zingiber officinale)

13. Zingiberaceae

14. Density Functional Theory (DFT)

15. Galangal (Alpinia officinarum)
7. Liquorice (Glycyrrhiza glabra L.)

8. Glycyrrhiza

9. Radix Astragali

10. Patrinia Herb 
جدول ا. كياهان مؤثر بر ويروسهاي خانواده كروناويروس

\begin{tabular}{|c|c|c|c|c|}
\hline شماره منابع & ماده مؤثره & ويروس هاى تحت تأثير & نام علمى كياه & نام قارسى كياه \\
\hline$[\mid \varphi-I V]$ & كليسيريزيك اسيد & ويروس سارس & Glycyrrhiza glabra L. & شيرينبيان \\
\hline$\left[r_{*}\right]$ & 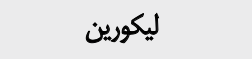 & ويروس سارس & Artemisia sp. & 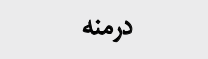 \\
\hline 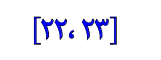 & مشابه داروهاي كلروكين & كوويد 19 & Zingiber officinale & 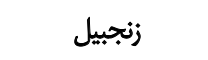 \\
\hline$[\Upsilon \omega-T \gamma]$ & فل فلاواثول & كويد 19 & Alpinia officinarum & خولنجان \\
\hline$[r \cdot, \mu)]$ & 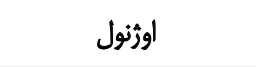 & كوويد 19 & Cinnamomum zeylanicum & دارجين \\
\hline$[\pi]$ & نيكليدين، آلفا-هيدرين & كوويد 19 & Nigella sativa $L$. & سياهلانه \\
\hline 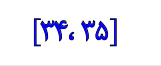 & فلاوتوئيد & كوويد 19 & Citrus paradise & كريبفروت (يوست) \\
\hline$[\mathrm{me}, \mathrm{MV}]$ & كليسيريزين & سارس & Laurus nobilis $L$. & برى بو \\
\hline$[\mathrm{ma}]$ & هترويلىساكاريدها & كوويد 19، سارس & Echinacea purpurea & 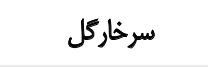 \\
\hline$[\tilde{\mu r}]$ & جينسنوسايد & "RSV & Panax quinquefollus & جينسينى \\
\hline
\end{tabular}

كه داراي خواص ضدآنفولانزايى بالايي هستند [•r"]. با توجه به

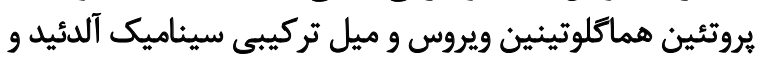

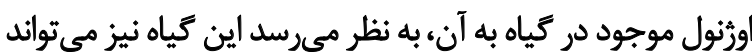

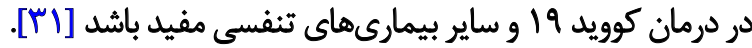

\section{سياهد|نdivi}

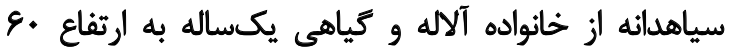

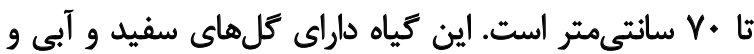

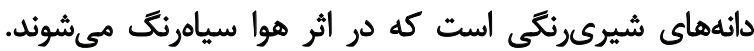

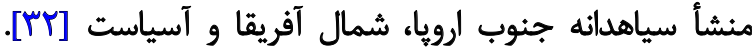

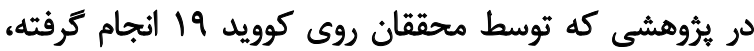

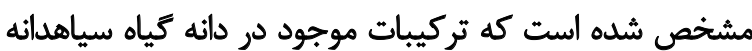

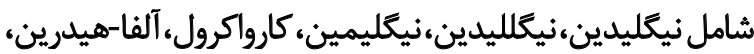

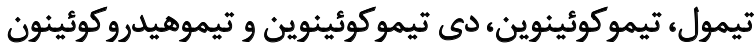

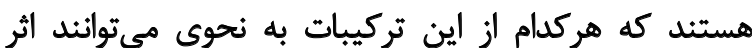

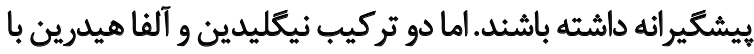

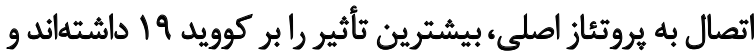

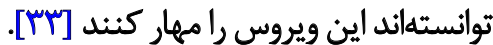

$$
\text { يوست تكريبفروته1 }
$$

كريڤفروت كياهى به صورت درخت و از خانواده مركبات

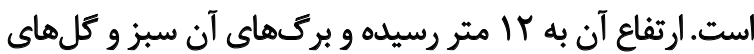

17.Fennel flower (Nigella sativa L.)

18. Grapefruit (Citrus paradise)
ويروس در موشها شده است، به كونهاى كه با كذشت دو تادئ

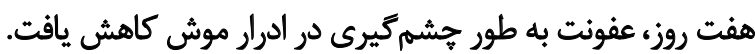

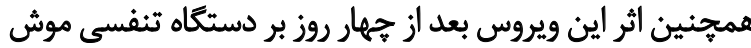

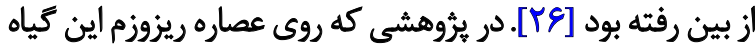

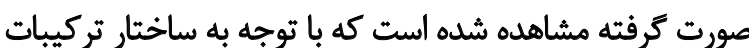

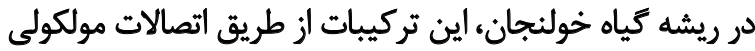

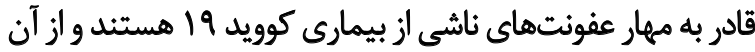

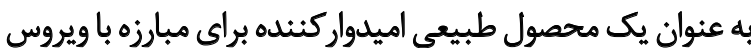
كويد 19 نام بردهاند [TrV]

19.0 of ols

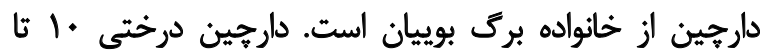

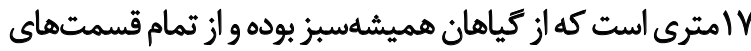

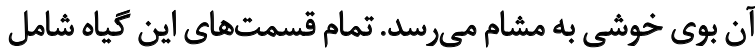

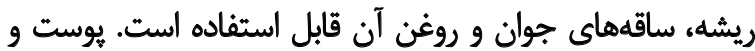

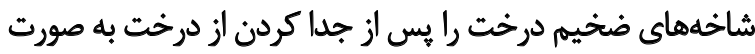

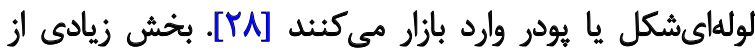

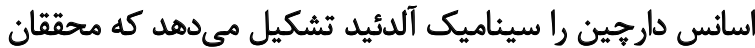

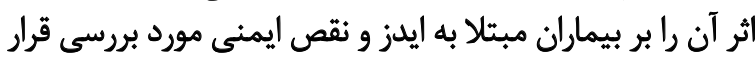

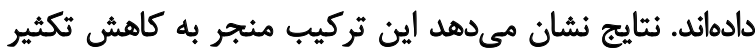

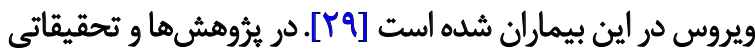

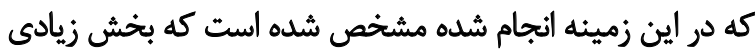

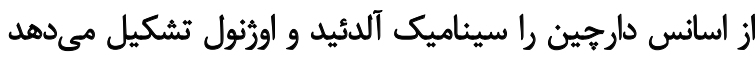

16. Cinnamon (Cinnamomum zeylanicum) 
جدول r. كياهان معرفىشده به عنوان ثقويتكنيده سيستم ايمنى در برابر ويروسها

\begin{tabular}{|c|c|c|}
\hline شماره منابع & نام علمى كياه & كياهان \\
\hline$[r q, r \cdot]$ & Sambucus nigra & آقطى \\
\hline$[\mathrm{er}]$ & Panax quinquefollus & جينسينك \\
\hline$[P f, Y A]$ & Aloe vera & ألوئلورا \\
\hline$[m, 4,4]$ & Astragalus memberanaceus & كون \\
\hline$[1 ., 810]$ & Zataria multiflora & آويشن شيرازى \\
\hline
\end{tabular}

ويروس سارس و همجنين قدرت مهاركنيدكي ويروس كوويد

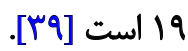

\section{كياهان تقويت كنثنده سيستم اليمنب}

فهرست كياهان تقويت كنيده سيستم ايمنى در برابر ويروسهادر جلدول شماره Y آورده شده است تقويت

$$
\text { rآ }
$$

آقطى سياه به خانواده كايريفولياسه ب تعلق دارد. از تركيبات

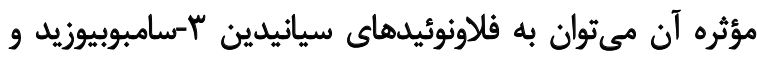

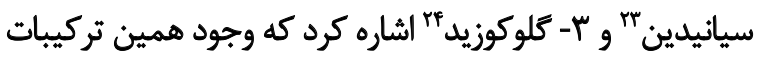
در عصارهاين كياه موجب افزايش سيتوكين ها به وسيله مونوسيتها

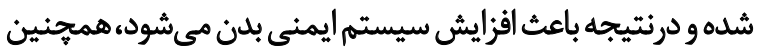

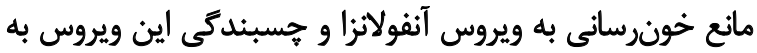

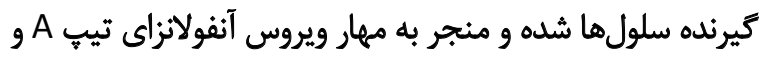

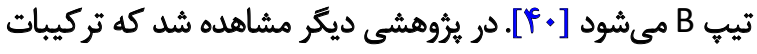

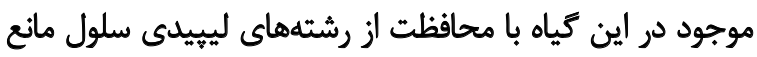

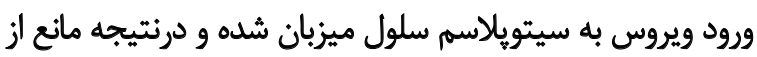

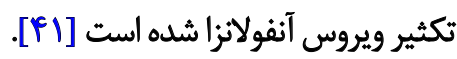

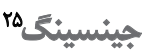

جينسينگ متعلق به خانواده آرلياسيه بوده و داراى يازده گونه

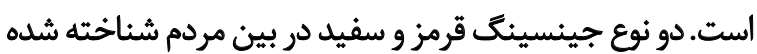

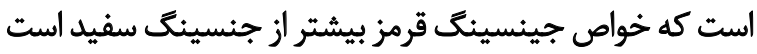

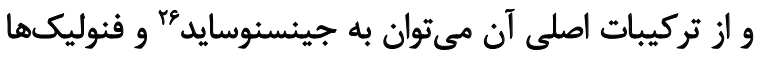

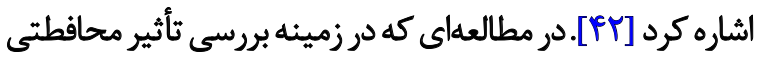

21. Elder (Sambucus nigra)

22. Caprifoliaceae

23. Cyanidin 3-sambubioside

24. 3-glucoside

25. Ginseng (Panax quinquefollus)

26. Ginsnocide

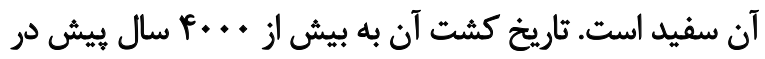
هند و مالزى ميرسد، اما در حال حاضر آمريكا حدود

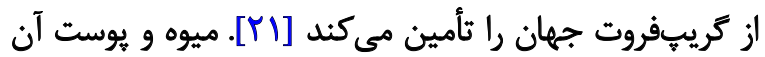
داراي تركيباتي همجون اسيدهاي هيوه، فلاونوئيدها، تركيبات

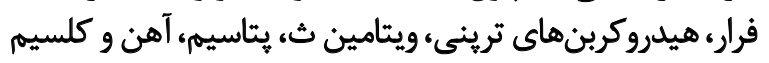

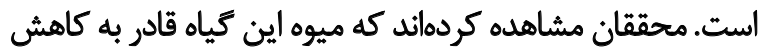

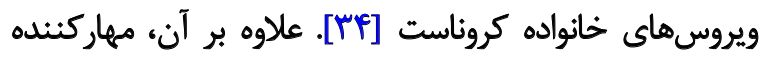

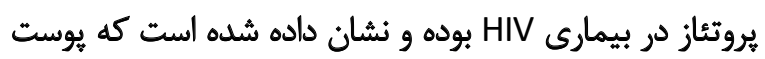

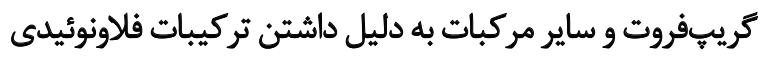

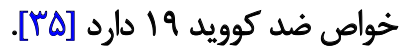

$$
19 \text {. }
$$

برك بو از خائواده برك بوييان با بركهاى معطر است كه ميوه

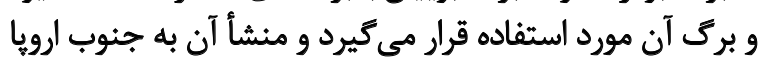
و آسياى صغير برمى

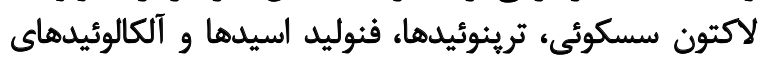

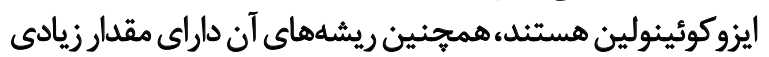

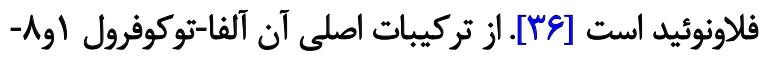

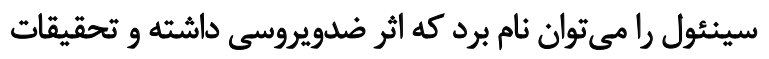

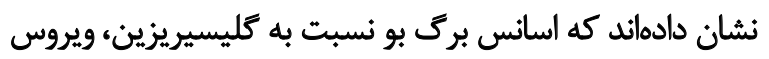
سارس را بهتر مهار مي كند [MV]

$$
\text { r. } 15,6,0
$$

سرخاركل متعلق به خانواده آفتابكردانان است. عصاره

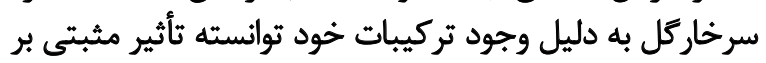

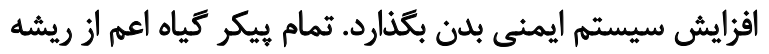

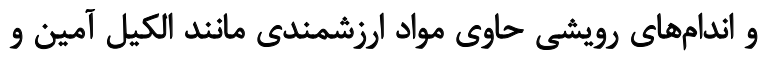

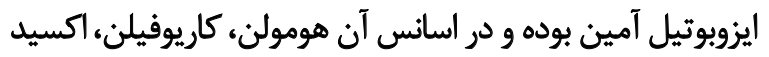

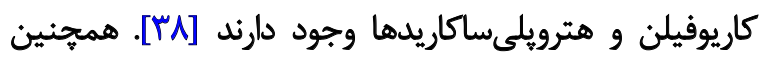
عصاره سرخارگل داراى خواص آنتيويروس آنفولاتزا

19. Bay laurel (Laurus nobilis L.)

20. Papule coneflower (Echinacea purpurea) 


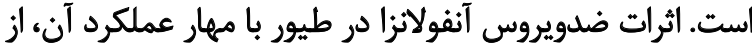

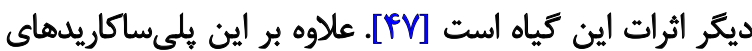

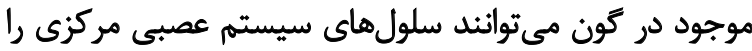

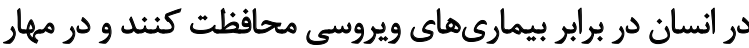

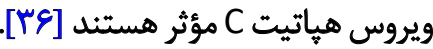

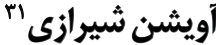

آويشن يكى از اعضاى خانواده نعناعيان است كه حدود دو تا

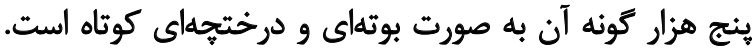

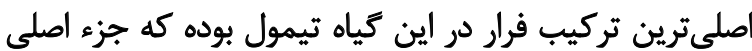

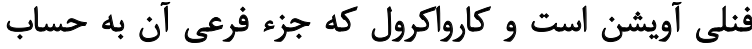

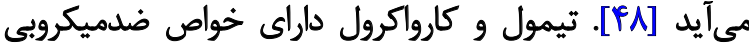

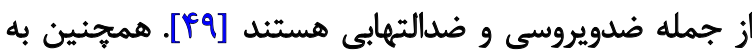

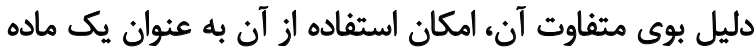

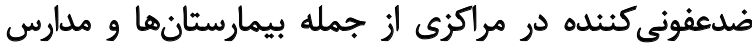

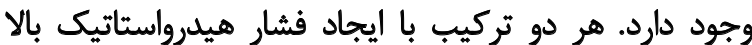

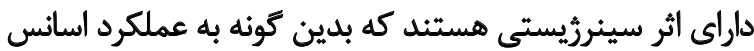

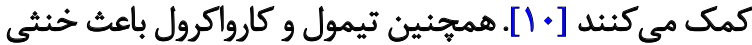

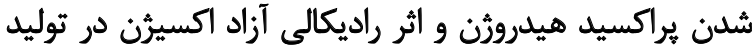

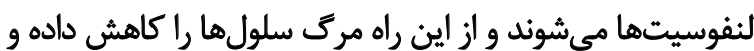
اثرات حفاظتي بر DNA اعمال مي كنيند و تضعيف سيستم ايمنى إنى

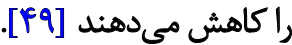

\section{نتيجليرى}

با توجه به اينكه يكى از راهكارهاي مؤثر در بيشكيرى و كنترل

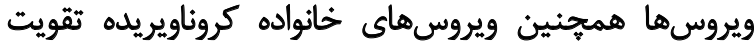

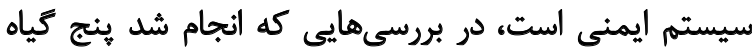

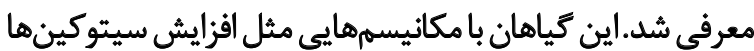

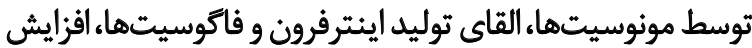

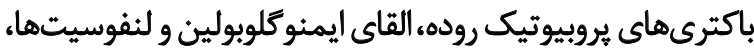

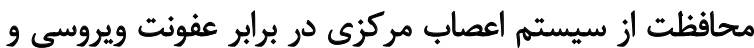

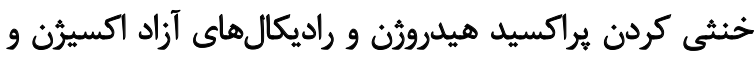

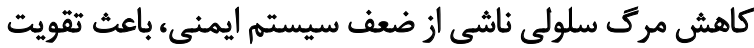

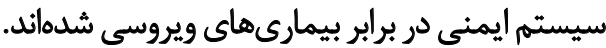

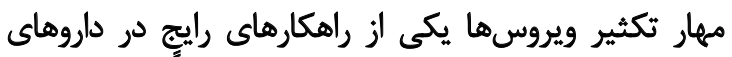

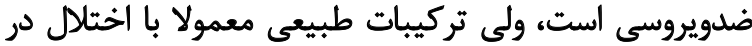

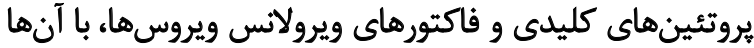

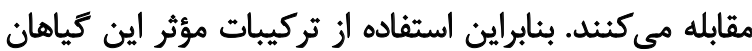

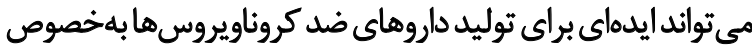

30. Thymes (Thymus vulgaris)

31. Zataria multiflora

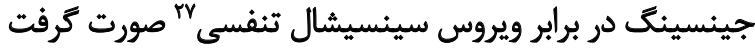

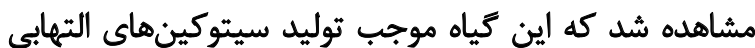

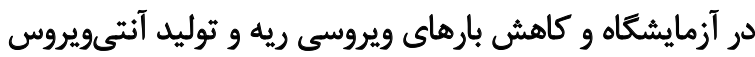

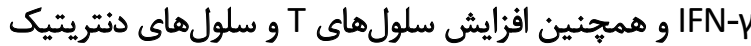

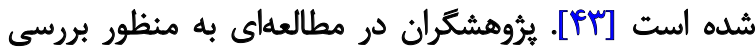

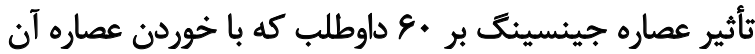
و خون كيرى در هفته جهارم و هفته هشته انجام شده، مشاهده

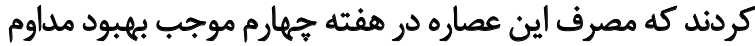

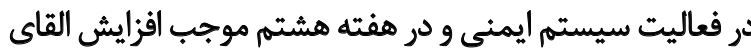

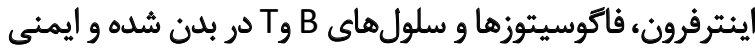

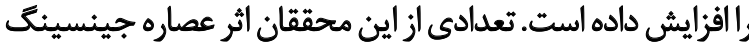

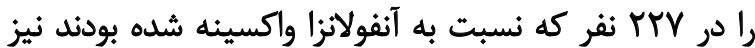

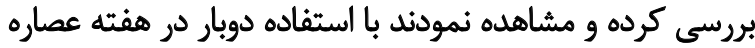

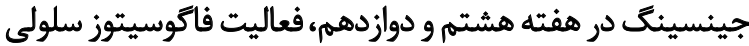

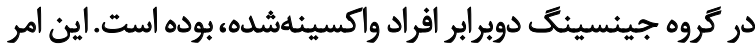

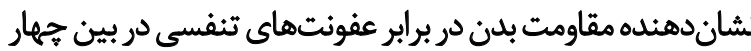

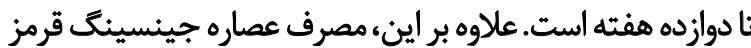

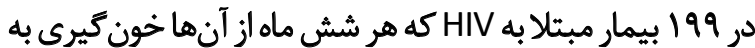

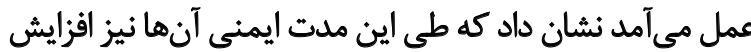

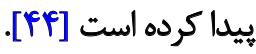

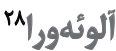

آلوئهورا يا صبر زرد و نامهاى ديكرى مانند شاخ بزى، كل

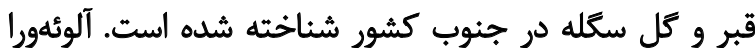

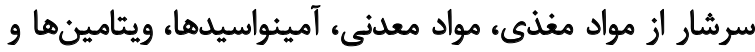

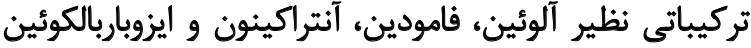

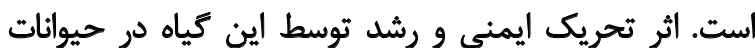

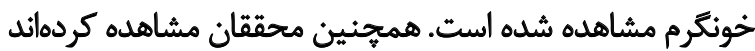

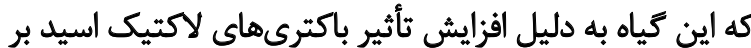

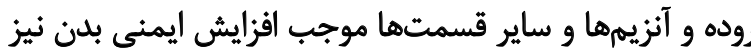

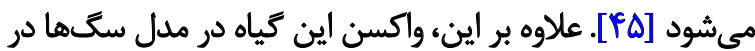

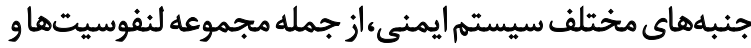

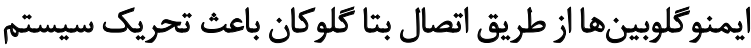

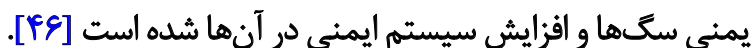

rqu

كون متعلق به خانواده باقلاييان است. اين كياه بومى آمريكا

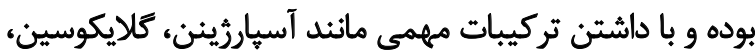

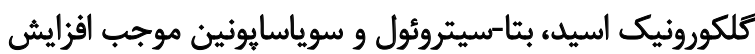

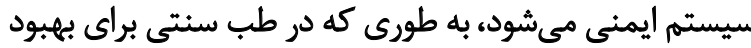

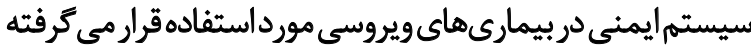

27. Respiratory Syncytial Virus (RSV)

28. Aloe vera

29. Milkvetch (Astragalus membranaceus) 
كوويد 19 باشد كه در سالهاي اخير و در اين روزها باعث مرى

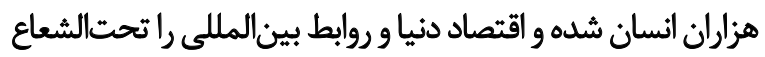

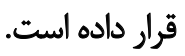

مالاحظات اخلاقى تر

بيروى أز اصول اخلاق بُروهش مقاله به صورت مرورى و بر اساس بررسى مقالات بوده است و شركتكننده و كد اخلاقى نداشته است.

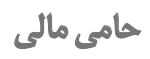
اين مقاله مرورى حامى مالى نداشته است. مشاركت ثويسندنًان

نويسندكان در تمامى مراحل نكارش به يك اندازه مشاركت

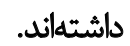

$$
\text { تعارض مثاقع }
$$

بنابر اظهار نويسندكان، اين مقاله تعارض منافع ندارد.

$$
\text { تشكر و قدردانى }
$$

نويسندكان از دانشكاه آزاد اسلامى واحد شيراز كمال تشكر را دارند. 


\section{References}

[1] Chyad Al-Noaemi M, Chyad Hammoodi AHM. COVID-19 and hydroxychloroquine relationship in the past, present, and future. The Pharma Innovation Journal. 2020; 9(4):944-8. [DOI:10.13140/ RG.2.2.28251.72487]

[2] Rezabakhsh A, Ala AR, Hassanpour Khodaei S. Novel Coronavirus (COVID-19): A new emerging pandemic threat. Journal of Research in Clinical Medicine (JRCM). 2020; 8(1):5. [DOI:10.34172/ jrcm.2020.005]

[3] Keyaerts E, Vijgen L, Pannecouque C, Van Damme E, Peumans $\mathrm{W}$, Egberink $\mathrm{H}$, et al. Plant lectins are potent inhibitors of coronaviruses by interfering with two targets in the viral replication cycle. Antiviral Research. 2007; 75:179-87. [DOI:10.1016/j.antiviral.2007.03.003] [PMID] [PMCID]

[4] Yang JL, Quy Ha TK, Oh WK. Discovery of inhibitory materials against PEDV corona virus from medicinal plants. Discovery of Japanese Journal of Veterinary Research. 64(Suppl 1):S53-S63. https://eprints. lib.hokudai.ac.jp/dspace/bitstream/2115/61017/1/09_Jun-Li\%20 Yang.pdf

[5] Khaerunnisa S, Kurniawan H, Awaluddin R, Suhartati S, Soetjipto S. Potential inhibitor of COVID-19 Main Protease $\left(\mathrm{M}^{\text {pro }}\right)$ from several medicinal plant compounds by molecular docking study. Pharmacology \& Toxicology. 2020; Preprints:2020030226. [DOI:10.20944/ preprints202003.0226.v1.]

[6] Dudani T, Saraogi A. Use of herbal medicines on Coronavirus. Acta Scientific Pharmaceutical Sciences. 2020; 4(4):61-3. [DOI:10.31080/ ASPS.2020.04.0518]

[7] Papari Moghadam Fard M, Ketabchi S, Farjam MH. Chemical composition, antimicrobial and antioxidant potential of essential oil of Ziziphus spina-christ var aucheri grown wild in Iran. Journal of Medicinal Plants and By-product. 2020; 9(Special):69-73. [DOI:10.22092/ JMPB.2020.121752]

[8] Mani JS, Johnson BJ, Steel JC, Broszczak DA, Neilsen PM. Natural product-derived phytochemicals as potential agents against coronaviruses: A review. Virus Research. 2020; 284:197989. [DOI:10.1016/j. virusres.2020.197989] [PMID] [PMCID]

[9] Reichling J, Schnitzler P, Suschke U, Saller R. Essential oils of aromatic plants with antibacterial, antifungal, antiviral, and cytotoxic properties: An overview. Forschende Komplementärmedizin (2006). 2009; 16(2):79-90. [DOI:10.1159/000207196] [PMID]

[10] Shabkhiz MA, Eikani MH, Golmohammad F, Bashiri Sadr Z. [Optimized pressurized hot water extraction of glycyrrhizic acid from $\mathrm{Li}$ corice roots (Persian)]. Innovative Food Technologies. 2015; 2(4):1121. [DOI: $10.22104 / J I F T .2015 .200]$

[11] Bae Ryu Y, Jae Jeong H, Hoon Kim J, Min Kim Y, Park JY, Kim D, et al. Biflavonoids from Torreya nucifera displaying SARS-CoV $3 \mathrm{CL}^{\text {pro }}$ inhibition. Bioorganic \& Medicinal Chemistry. 2010; 18(22):7940-7. [DOI:10.1016/j.bmc.2010.09.035] [PMID] [PMCID]

[12] Aanouz I, Belhassan A, El-Khatabi K, Lakhlifi T, El-Idrissi M, Bouachrine M. Moroccan Medicinal plants as inhibitors against SARS-CoV-2 main protease: Computational investigations. Journal of Biomolecular Structure \& Dynamics. 2020; 1-9. [DOI:10.1080/07391 102.2020.1758790] [PMID] [PMCID]

[13] Ahmad A, Rehman MU, Alkharfy KM. An alternative approach to minimize the risk of Coronavirus (Covid-19) and similar infections. European Review for Medical and Pharmacological Sciences. 2020; 24(7):4030-4. https://www.europeanreview.org/article/20873
[14] Ketabchi S, Moatari A Shadram M, Rostami Y. The antiinfluenza virus activity of Anchusa italica. Asian Journal of Experimental Biological Sciences. 2011; 2(4):758-61. https:// www.ajebs.com/\#: :text=ASIAN\%20JOURNAL\%200F\%20EX PERIMENTAL\%20BIOLOGICAL\%20SCIENCES\%20is\%20a\%20 peer\%2Dreviewed,freely\%20available\%20to\%20researchers $\% 20$ worldwide.

[15] Kozhuharova A, Stanilova M. In vitro cultures initiation from seeds of Bulgarian localities of Glycyrrhiza glabra L. (Fabaceae). Journal of BioScience \& Biotechnology. 2017; (Special):25-30. https://web.a.ebscohost. com/abstract?direct=true\&profile=ehost \&scope=site\&authtype=crawl er\&jrnl=1314623

[16] Shen XP, Xiao PG, Liu CX. Research and application of Radix Glycyrrhizae. Asian Journal of Pharmacodynamics and Pharmacokinetics. 2007; 7(3):181-200. https://www.researchgate.net/publication/228473512 Research_and_application_of_Radix_Glycyrrhizae

[17] Dehghani K, Hamidi F, Moheb Aliyan H. [Investigating the Preparation of Glycyrrhiza glabra L. Products and its Applications in industry (Persian)]. Paper presented at: Second International Conference on Medicinal Plants, Organic Agriculture, Natural Resources and Pharmaceuticals. 13 March 2019; Mashhad, Iran. https://www.en.symposia.ir/ CONFMTO2

[18] Papari Moghadam Fard M, Ketabchi S. [Natural ingredients of native plants of Iran: Along with the introduction of identification devices and methods (Persian)]. Shiraz: Takht Jamshid Publication; 2017. https:// www.gisoom.com/book/11381701/\%DA\%A9\%D8\%AA\%D8\%

[19] Mohabatkar H, Nosrati M, Behbahani M, Rahiminejad MR. Antibac terial and mutagenicity activity of different species of artimisia spp. and their effect on proliferation of human lymphocytes. Journal of Mazandaran University of Medical Sciences. 2016; 26(142):82-95. https://www.sid.ir/fa/journal/ViewPaper.aspx?ID=276926

[20] Li SY, Chen C, Zhang HQ, Guo HY, Wang H, Wang L, et al. Identification of natural compounds with antiviral activities against SARS-associated coronavirus. Antiviral Research. 2005; 67:18-23. [DOI:10.1016/j.antiviral.2005.02.007] [PMID] [PMCID]

[21] Papari Moghadam Fard M, Fakhri A. [Natural and healing properties of monocotyledonous and dicotyledonous plants (Persian)]. Shiraz: Takhtjamshid; 2016. https://www.gisoom.com/book/11381698/\%DA\%A9\% D8\%AA\%D8\%A7\%D8\%A8-\%D8\%AE\%D9\%88\%D8\%A7\%D8\%B5-

[22] Kumar Srivastava AK, Kumar A, Misra N. On the inhibition of COVID-19 protease by Indian herbal plants: An in silico investigation. arXiv preprint arXiv:2004.03411. 2004; 1-14. https://arxiv.org/abs/2004.03411.

[23] Rathinavel T, Palanisamy M, Palanisamy S, Subramanian A, Thangaswamy S. Phytochemical 6-Gingerol: A promising drug of choice for COV ID-19. International Journal of Advanced Science and Engineering. 2020 6(4):1482-9. [DOI:10.29294/IJASE.6.4.2020.1482-1489]

[24] Alasmary AF, Assirey AE, El-Meligy MR, Awaad A, El-sawaf AL, Allah $\mathrm{MM}$, et al. Analysis of Alpina officinarum Hance, chemically and biologically. Saudi Pharmaceutical Journal (SPJ). 2019; 27(8):1107-12. [DOI:10.1016/j.jsps.2019.09.007] [PMID] [PMCID]

[25] Zakaryan H, Arabyan E, Oo A, Zandi K. Flavonoids: Promising natural compounds against viral infections. Archives of Virology. 2017; 162:(9):2539-51. [DOI:10.1007/s00705-017-3417-y] [PMID] [PMCID]

[26] Sawamura R, Shimizu T, Sun Y, Yasukawa K, Miura M, Toriyama M, et al. In vitro and in vivo anti-influenza virus activity of diaryl heptanoids isolated from Alpinia officinarum. Antiviral Chemistry and Chemotherapy. 2010; 21(1):33-41. [DOI:10.3851/IMP1676] [PMID] 
[27] Goswami D, Kumar MK. Ghosh S, Das A. Natural product compounds in Alpinia officinarum and ginger are potent SARS-CoV-2 papain-like protease inhibitors. ChemRxiv. Preprint. 2020; 4. [Online Publishing]. [DOI:10.26434/chemrxiv.120711997.vl]

[28] Ahmadi R, Ghasemi N. [Effect of local application and injection of cinnamomum zeylanicum on burn wound improvement in diabetic and non-diabetic male rats (Persian)]. Medical Sciences Journal of Islamic Azad Universit. 2015; 25(1):27-32. http://tmuj. iautmu.ac.ir/article-1-901-fa.html

[29] Liu L, Wei FX, Qu ZY, Wang SQ, Chen G, Gao H, et al. The antiadenovirus activities of cinnamaldehyde in vitro. Laboratory Medicine. 2009; 40(11):669-74. [DOI:10.1309/LMF0U47XNDKBZTRQ]

[30] Vimalanathan S, Hudson J. Anti-influenza virus activity of essential oils and vapors. American Journal of Essential Oils and Natural Products. 2014; 2(1):47-53. https://www.researchgate. net/publication/267035381_Anti-influenza_virus_activity_of_essential_oils_and_vapors

[31] Da Silva RJK, Baia Figueiredo BL, G byler K, Nsetzer W. Essential oils as antiviral agents, potential of essential oils to treat SARS-CoV-2 Infection: An in-silico investigation. International Journal of Molecular Sciences. 2020; 21(10):3426. [DOI:10.3390/ ijms21103426] [PMID] [PMCID]

[32] Gholinezhad E, Rezaei Chiyaneh E. [Evaluation of grain yield and quality of black cumin (Nigella sativa L.) in intercropping with chickpea (Cicer arietinum L.) (Persian)]. Iranian Journal of Crop Sciences. 2014; 16(3):236-49. http://agrobreedjournal.ir/browse. php?a_code=A-10-1-13\&sid=1\&slc_lang=fa

[33] Bouchentouf S, Missoum N. Identification of Compounds from Nigella Sativa as new potential inhibitors of 2019 novel Coronasvirus (Covid-19): Molecular docking study [Internet]. 2020. [Updated 31 March 2020]. Available from: https://chemrxiv.org/articles/ preprint/Identification_of_Compounds_from_

[34] Cheng L, Zheng W, Li M, Huang J, Bao S, Xu Q, et al. Citrus fruits are rich in flavonoids for immunoregulation and potential targeting ACE2 [Internet]. 2020. [Updated 23 February 2020]. Available from: https://www.preprints.org/manuscript/202002.0313/v1

[35] Patrakar R, Mansuriya M, Patil P. Phytochemical and pharmacological review on Laurus Nobilis. International Journal of Pharma and Bio Sciences. 2012; 1(2):595-602. https://www.semanticscholar.org/paper/Phytochemical-and-Pharmacological-Reviewon-Laurus-Patrakar-Mansuriya/1cd15010e12589a567edbcbde5 9a203502689794

[36] Sefidkan F. Can medicines with antiviral activities be made from medicinal plants? Iran Nature. 2020; 5(2):5-13. https://irannature.areeo. ac.ir/article_121626_31d7d15bd2f9b3b7c226be81f40ca6ff.pdf

[37] Hudson BJ. Applications of the phytomedicine echinacea purpurea (purple coneflower) in infectious diseases. Journal of Biomedicine \& Biotechnology. 2012; 2012:769896. [DOI:10.1155/2012/769896] [PMID] [PMID]

[38] Anandan R, Suseendran G, Zaman N, Nawaz Brohi S, Duraisamy B Deepak BS. Echinacea purpurea to treat Novel Coronavirus (2019nCoV). TechRxiv. 2020; 1-7. [DOI:10.36227/techrxiv.12115596.v2.]

[39] Farshi P, Ceren Kaya E, Hashempour-Baltorkb F, Khosravi-Darani $\mathrm{K}$. A comprehensive review on the effect of plant metabolites on coronaviruses: Focusing on their molecular docking score and IC50 values. Preprints [Internet]. 2020 [Updated 18 May 2020]. Available from: https://www.preprints.org/manuscript/202005.0295/v1
[40] Zakey-Rones Z, Thom E, Wollan T, Wadstein J. Randomized study of the efficacy and safety of oral elderberry extract in the treatment of influenza A and B virus infections. The Journal of International Medical Research. 2004; 32(2):132-40. [DOI:10.1177/1473 23000403200205] [PMID]

[41] Narayan Mishra J, Kumar Verma N. An Overview on Panax ginseng.International Journal of Pharma And Chemical Research. 2017; 3(3):516-22. http://ijpacr.com/files/21-07-2017/17.pdf

[42] Hosseini SH, Amoghli Tabrizi B, Mazlom Mogaddam SSR. Evaluation at ginseng on lipid profiles, liver and renal markers in diabetic rats. Journal of Advances in Medical and Biomedical Research. 2011; 19(75):11-7. http://zums.ac.ir/journal/article-1-1487-en. html

[43] Radad K, Gille G, Rausch WD. Use of ginseng in medicine: Perspectives on CNS disorders. Iranian Journal of Pharmacology and Therapeutics (IJPT). 2004; 3(2):30-40. https://www.sid.ir/en/journal/ViewPaper.aspx $\mathbf{i d}=34624$

[44] Bazari Moghaddam S, Haghighi M, Sharif Rohani M, Hamidi M, Ghasemi M. Effects of Aloe vera extract on growth indices, carcass composition and bacterial flora of intestine in Siberian sturgeon (Acipenser Baerii). Iranian Journal of Fisheries Sciences. 2016; 25(1):1-5. http://isfj.ir/article-1-1551-fa.htm

[45] Altug N, Yuksek N, Tevfik Agaogle Z. Immunostimulatory effects of Aloe vera and $\beta$-Glucan on cellular and humoral immune re sponses following vaccination with polyvalent vaccines in dogs. Vetdergikafkas. 2010; 16(3):405-12. https://www.researchgate. net/publication/283857458 Immunostimulatory effects of aloe_vera_and_b-glucan_on_cellular_and_humoral_immune responses_following_vaccination_with_polyvalent_vaccines_in_ dogs

[46] Majeed Khan H, Raza SM, Ahmad Anjum A, Ali MA, Akbar H. Antiviral, embryo toxic and cytotoxic activities of Astragalus membranaceus root extracts. Pakistan Journal of Pharmaceutical Sciences. 2019; 32(1):137-42. [PMID]

[47] Ahmadi R, Alizadeh A, Ketabchi S. Antimicrobial activity of the essential oil of Thymus kotschyanus grown wild in Iran. International Journal of Biosciences (IJB). 2015; 3(6):239-48. [DOI:10.12692/ ijb/6.3.239-248]

[48] Purnavab S, Ketabchi S, Rowshan V. Chemical composition and antibacterial activity of methanolic extract and essential oil of Iranian Teucrium Polium against some of phytobacteria. Natural Product Research. 2015; 29(14):1376-9. [DOI:10.1080/14786419 2014.1000320] [PMID]

[49] Kaeidi A, Rahmani MR, Hasanshahi J. The protective effect of carvacrol and thymol as main polyphenolic compounds of thyme on some biologic systems in disease condition: A narrative review. Journal of Rafsanjan University of Medical Sciences. 2020; 19(1):81-96. [DOI:10.29252/jrums.19.1.81] 